DOI 10.37882/2223-2982.2021.03.11

\title{
ПРОБЛЕМЫ ВЗАИМОДЕЙСТВИЯ СПОРТСМЕНА И ЦИФРОВЫХ ИНТЕРФЕЙСОВ УСТРОЙСТВ И ПРИЛОЖЕНИЙ ДЛЯ ТРЕНИРОВКИ БЕГА
}

\section{PROBLEMS OF INTERACTIONS OF THE ATHLETE AND DIGITAL INTERFACES OF DEVICES AND APPLICATIONS FOR RUNNING TRAINING \\ D. Herzen}

Summary: Running has become a popular sport in recent years. With the advancement of digital technology, there has been an increase in the supply and demand for equipment to support runners during training. At the same time, there is a lack of attention of researchers and developers to the problem of interaction of an athlete with a digital interface. In this article, based on the results of a survey of athletes, expert assessments of the prospects for organizing the training process using digital technologies, and analysis of scientific literature, two vectors of the design of the interface of digital technologies for running training were identified: a shift in emphasis from effective indicators to indicators related to running technique, and a change in feedback algorithms. The article also presents an analysis of the problems associated with improving the digital interfaces of devices and applications for running training. The first problem relates to the interaction of the athlete with the digital interface of devices and running training applications. The second problem, which organically follows from the first, is what information should be transmitted to the athlete. The third problem is related to the timing of providing information about running technique to an athlete. We believe that taking into account and solving these problems will help improve the training process of athletes.

Keywords: sport, running, digital technologies, training process, running technique.

\author{
Герцен Дмитрий Александрович \\ Аспирант, ГАОУ ВО г. Москвы «Московский городской \\ педагогический университет» \\ gertsen.dmitry@gmail.com
}

Аннотация: Бег стал популярным видом спорта в последние годы. С развитием цифровых технологий отмечается повышение спроса и предложения на оборудование, поддерживающее бегунов во время тренировок. Вместе с тем, отмечается дефицит внимания исследователей и разработчиков проблеме взаимодействия спортсмена с цифровым интерфейсом. В данной статье на основе результатов опроса спортсменов, экспертных оценок перспектив организации тренировочного процесса с использованием цифровых технологий, анализа научной литературы были определены два вектора дизайна интерфейса цифровых технологий для тренировки бега: смещение акцентов с результативных показателей на показатели, относящиеся к технике бега, и изменение алгоритмов обратной связи. Также в статье представлен анализ проблем, связанных с совершенствованием цифровых интерфейсов устройств и приложений для тренировки бега. Первая проблема связана со взаимодействием спортсмена с цифровым интерфейсом устройств и приложений для тренировки бега. Вторая проблема, которая органически вытекает из первой, заключается в том, какая информация должна передаваться спортсмену. Третья проблема связана с определением времени предоставления информации о технике бега спортсмену. Мы считаем, что учет и решение данных проблем будут способствовать улучшению тренировочного процесса спортсменов.

Ключевые слова: спорт, бег, цифровые технологии, тренировочный процесс, техника бега.

зуются марафонцы сегодня в процессе тренировки.

В спортивных кругах существует и противоположное мнение. Многие бегуны на длинные и сверхдлинные дистанции ограничивают использование цифровых технологий на тренировках и отказываются от них во время марафона. Причина заключается в том, что применение специальных устройств и оборудования в беге может отвлекать спортсмена от собственного барометра. Марафонцы-минималисты используют низкотехнологичную альтернативу: ламинированную полоску бумаги, используемую как браслет, с указанием времени, которое бегун должен пройти на каждом километре.

Некоторые тренеры и спортсмены не рекомендуют 
использовать наушники, потому что музыка во время тренировки и марафона может излишне мотивировать бегуна, вырывать из контекста соревнования. Существует и противоположная тактика. Наушники и возможность трансляции через них звукового сигнала применяются для улучшения техники бега. Согласно исследованиям В.Н. Коновалова, А.С. Максимова, И.А. Фатьянова наиболее эффективной тактикой бега на марафонскую дистанцию является бег короткими быстрыми шагами (около 180 в минуту) $[3,4,5]$. Поэтому некоторые бегуны загружают тиканье метронома в плееры, чтобы поддерживать необходимый темп.

В 2000 году компания Garmin выпустила спортивные часы, которые отслеживают пройденное расстояние, средний темп и другие параметры. Сегодня основное внимание в цифровых интерфейсах для тренировки бега уделяется темпу, расстоянию, маршруту и частоте сердечных сокращений. В последнее время цифровые технологии стали использовать кинематические показатели, такие как траектория касания ступней бегуна поверхности, длина и частота шагов, поза бегуна и т.д. M.M. Jensen, F.F. Mueller считают, что включение данных показателей в цифровые интерфейсы современных устройств позволит положительно влиять в тренировочном процессе на экономичность бега, снизить риски травмирования спортсменов и, в конечном итоге, окажет существенное воздействие на результат бега [12].

Вместе с тем, отмечается дефицит внимания исследователей и разработчиков проблеме взаимодействия спортсмена с цифровым интерфейсом [1, 2].

\section{Методы}

Начиная с 2000 года в продаже появилось несколько часов, специально предназначенных для бегунов, например, Garmin, Polar и Suunto, использующих различные технологии, такие как GPS, мониторы сердечного ритма и акселерометры. Эти устройства можно использовать для сбора различной информации о тренировочном процессе, например, темпа, времени, частоты пульса и расстояния. Информация, как правило, предоставляется пользователю либо на дисплее часов во время бега, либо в виде подробного обзора после тренировки. Приложения RunKeeper и Endomondo адаптировали концепцию спортивных часов, используя встроенный в смартфон GPS-трекер и акселерометр для отслеживания результатов бегуна и представления данных на экране телефона или на веб-сайте.

Кроме того, в спортивные часы для бега и приложения разработчики стали интегрировать расписания тренировок, механизмы, позволяющие корректировать технику бега, например, спортсмен предупреждается, если его темп отклоняется от заданного тренировочным сеансом.
И спортивные часы, и приложения для бега стали популярными среди бегунов любого уровня подготовки. Однако в этих технологиях часто основное внимание уделяется беговой производительности, например, времени и расстоянию, и меньше внимания технике бега (C.A. Clermont, L. Duffett-Leger, B.A. Hettinga, R. Ferber [9]).

Последние достижения в области датчиков и носимых устройств сделали возможным измерение различных параметров техники бега. Используя носимые датчики и технологии обнаружения движения на основе камеры, исследователи показали, что можно определить параметры техники, такие как тип удара стопой (FST), время контакта с землей (GCT), каденция, вертикальные колебания (VO) и сгибание-разгибание колена $[6,7,8]$, что позволяет профилировать стили бега, определять возможности для улучшений и распознавать показатели усталости. Однако параметры, связанные с техникой, измеренные датчиками, в первую очередь становятся доступными для бегунов после тренировки, часто в виде кинематического анализа. Таким образом, поскольку обратная связь недоступна во время тренировки, бегунам трудно соответствующим образом корректировать технику бега во время тренировочного процесса. Несмотря на то, что некоторые технологии определяют параметры, связанные с техникой, в режиме реального времени, существуют ограничения по использованию информации для создания вспомогательных интерфейсов.

Современные часы для бега, например, Garmin Forerunner 620, также предлагают информацию, относящуюся к технике бега, например, GCT, каденция и VO. B результате бегун может проверять эти параметры в виде чисел на часах во время бега. Однако мы обнаружили, что, используя эти часы, может быть трудно связать, понять и использовать эту информацию для улучшения техники бега. Кроме того, постоянное обращение к часам во время тренировки с целью наблюдения за эффектами корректировки движения нецелесообразно.

Для того, чтобы использовать параметры, связанные с техникой бега, и решить коммуникативные проблемы, необходимо исследовать альтернативные методы и механизмы обратной связи, которые отличаются от традиционных цифровых интерфейсов на основе экрана [12, 13]. Такой интерфейс представляет собой сенсорную систему, основанную

на тактильной обратной связи. Система предупреждает спортсменов, если их руки совершают нецелесообразные движения, которые могут повлиять на экономичность бега. Таким образом, система действует как универсальный тренер по бегу, предоставляя обратную связь по части техники бега в режиме реального времени [12].

M. Eriksson, R. Bresin предлагают использовать слухо- 
вую обратную связь, чтобы предупреждать бегунов во время бега, если их вертикальное смещение превышает заданное целевое значение [10]. Вертикальное смещение относится к работе, которую бегун должен выполнять против силы тяжести на каждом шаге. Следовательно, малое вертикальное смещение должно привести к повышению экономичности бега, поскольку энергия используется для перемещения бегуна вперед, а не вверх. Носки и стельки Sensoria Fitness Socks - это коммерческий продукт, в котором используются чувствительные к давлению носки с измерениями в приложении для смартфона (Sensoria Fitness). Затем приложение использует слуховую обратную связь, чтобы предупреждать бегунов, если они отклоняются от заданных пользователем характеристик бега [8]. C. Strohrmann, J. Seiter, Y. Llorca, G. Tröster [13], M. Eriksson, R. Bresin [10] предлагают использовать системы оповещения, которые предупреждают бегунов, если движение нецелесообразно. Однако понять, как исправить ошибочное движение и в какой степени оно требует исправления, сложно на основе дискретной обратной связи.

На основе анализа научной литературы по теме был составлен опросник, нацеленный на выявление трудностей в организации тренировочного процесса бегунов с использованием различных цифровых технологий. В исследовании приняли участие 136 спортсменов-любителей. Опрос был проведен АНО ЦПСМ «Московский марафон» в 2021 году.

\section{Результаты}

На основе результатов проведенного опроса, экспертных оценок, анализа научной литературы были определены два вектора дизайна интерфейса цифровых технологий для тренировки бега: смещение акцентов с результативных показателей на показатели, относящиеся к технике бега, и изменение алгоритмов обратной связи.

Смещение акцентов предполагает переориентацию от технологий, ориентированных на производительность, до технологий, ориентированных на технику. М.М. Jensen, F.F. Mueller считают, что такие показатели, как $\mathrm{GCT}, \mathrm{VO}$, являются прямыми результатами техники бега и представляют собой эффекты физических движений, но не описания самого движения. Показателями, отражающими технику бега, являются FST и угол сгибанияразгибания колена, который описывает кинематическое движение бегуна [12].

Изменение алгоритмов обратной связи предполагает уход от репрезентативной обратной связи в сторону вспомогательной обратной связи. Репрезентативная обратная связь предоставляет бегуну информацию о беге в форме чисел. Контекстуальная репрезентативная обрат- ная связь относится к объединению различных данных, чтобы обеспечить спортсмену лучшее понимание предоставляемых данных, например, путем сопоставления карты маршрута с различными темпами.

Вспомогательная обратная связь в реальном времени ориентирована на передачу информации во время выполнения движений. Примеры включают системы, которые обеспечивают обратную связь с предупреждением и, таким образом, помогают бегуну обнаружить отклонение определенного показателя от ожидаемого, например, предупреждение часов для бега о снижении темпа или тактильный сигнал, обнаруживающий нецелесообразное движение руки [12]. Эти системы существуют как виртуальные тренеры в интерфейсах беговых тренировок, ориентированных на результат, например, Help2Run, TrainingPeaks, которые отслеживают график тренировок бегуна, адаптируют расписание с учетом результатов за неделю и постоянно помогают бегуну во время тренировок.

Таким образом, мы утверждаем, что необходимо исследовать, как предоставлять спортсмену комплексную обратную связь, связанную с техникой бега.

\section{Обсужкение}

В обсуждении данной проблемы мы выделяем три проблемы.

Первая проблема связана со взаимодействием спортсмена с цифровым интерфейсом устройств и приложений для тренировки бега. Использование экрана на спортивных часах в качестве интерактивной платформы для управления беговыми движениями может быть затруднительным, поэтому слуховые интерфейсы были приняты за основу в технологии обучения бегу. Однако, здесь также исследователи видят определенные трудности. Например, если сообщение представляет собой темп на последнем круге, звуковое сообщение кажется разумным, тогда как для информирования бегуна о том, поднялось ли его колено слишком высоко, более подходящим может быть тактильный сигнал [12]. Кроме того, некоторые ученые выступают за использование мультимодальной обратной связи в моторном обучении, поскольку она потенциально поддерживает одновременное обучение нескольким аспектам сложного движения [13].

Вторая проблема, которая органически вытекает из первой, заключается в том, какая информация должна передаваться спортсмену. Например, M.M. Jensen, F.F. Mueller отмечают, что большинство бегунов не обладают достаточными моториками, чтобы менять тип удара ногой с тыла на передний, просто получая информацию о том, где они приземляются, как это предоставляется 
SFS [12]. Вместо этого разработчики вспомогательных интерфейсов для тренировки бега должны изучать приемы у тренеров, которые рекомендуют бегунам «делать меньшие шаги» или «смотреть вниз в долину», чтобы изменить свой стиль бега.

Третья проблема связана с определением времени предоставления информации о технике бега спортсмену. Постоянное предоставление информации бегуну, вероятно, снижает удобство использования цифрового интерфейса. Периодическая информация, например, на каждом круге подходит для некоторых параметров, однако в других случаях бегуну может потребоваться немедленная обратная связь при изменении движения. Системы предупреждающей обратной связи помогают бегуну избегать нецелесообразных движений, однако получение только отрицательной обратной связи может демотивировать спортсменов.

\section{Зак^ючение}

В этой статье мы охарактеризовали направления разработок в области проектирования цифровых технологий для тренировки бега. Основываясь на современных цифровых технологиях, мы утверждаем, что существует необходимость исследовать, как создавать вспомогательные, ориентированные на технику бега цифровые интерфейсы для тренировки бега. Мы также выявили три проблемы, которые возникают при использовании цифровых технологий в процессе тренировки бегунов. Мы считаем, что учет и решение данных проблем будут способствовать улучшению тренировочного процесса спортсменов.

\section{ЛИТЕРАТУРА}

1. Егорова Н.М. Анализ возможностей применения мобильных технологий в сфере физической культуры и спорта / Н.М. Егорова // Физическая культура. Спорт. Туризм. Двигательная рекреация. - 2020. - Т. 5. - №1. - С. 153-158.

2. Кариаули А.С. Векторы использования социальных сетей и мобильных приложений в дисциплине «Физическая культура» / А.С. Кариаули // Физическая культура: воспитание, образование, тренировка. - 2019. - №1. - С. 51-54.

3. Коновалов В.Н. Построение этапа непосредственной предсоревновательной подготовки бегунов на сверхдлинные дистанции: дис. ... канд. пед. наук: 13.00.04 / В.Н. Коновалов. - Москва, 1986. - 151 с.

4. Максимов А.С. Особенности нормирования тренировочной нагрузки в системе подготовки бегунов на сверхдлинные дистанции: дис. ... канд. пед. наук: 13.00.04 / А. С. Максимов. - Москва, 1983. - 145 с.

5. Фатьянов И.А. Конвергентные стратегии повышения конкурентоспособности марафонцев: дис. ... канд. пед. наук: 13.00.04 / И.А. Фатьянов. - Волгоград, 2016. $-430 \mathrm{C}$.

6. Ahamed N.U., Benson L.C., Clermont C.A., Pohl A.J., Ferber R. New considerations for collecting biomechanical data using wearable sensors: How does inclination influence the number of runs needed to determine a stable running gait pattern? / N.U. Ahamed, L.C. Benson, C.A. Clermont, A.J. Pohl, R. Ferber // Sensors. 2019. - \#19(11). -2516.

7. Altman A.R., Davis I.S. A kinematic method for footstrike pattern detection in barefoot and shod runners / A.R. Altman, I.S. Davis // Gait Posture. - 2012. - \#35. P. 298-300.

8. Burns G.T., Deneweth Zendler, J., Zernicke R.F. Validation of a wireless shoe insole for ground reaction force measurement // G.T. Burns, J. Deneweth Zendler, R.F. Zernicke // Journal of Sport Science. - 2018. -\#1. - P. 1-10.

9. Clermont C.A., Duffett-Leger L., Hettinga B.A., Ferber R. Runners' Perspectives on 'Smart' wearable technology and its use for preventing injury / C.A. Clermont, L. Duffett-Leger, B.A. Hettinga, R. Ferber // International Journal of Human-Computer Interaction. - 2019. \#1. - P. 1-10.

10. Eriksson M., Bresin R. Improving running mechanics by use of interactive sonification. / M. Eriksson, R. Bresin // Interaction Sonification workshop (ISon). - 2010, P. 95-98.

11. Eskofier B.M., Musho E., Schlarb H. Pattern classification of foot strike type using body worn accelerometers. / B.M. Eskofier, E. Musho, H. Schlarb // IEEE International Conference on Body Sensor Networks. - BSN, 2013. - P. 1-4.

12. Jensen M.M., Mueller F.F. Running with technology: Where are we heading? // M.M. Jensen, F.F. Mueller. Proceedings of the 26th Australian Computer-Human Interaction Conference on Designing Futures: the Future of Design. - Sydney, 2014. - P. 527-530.

13. Strohrmann C., Seiter J., Llorca Y., Tröster G. Can Smartphones Help with Running Technique? / C. Strohrmann, J. Seiter, Y. Llorca, G. Tröster // Procedia Compuer Science. - 2013. - \#19. - P. 902-907.

(с Герцен Дмитрий Александрович (gertsen.dmitry@gmail.com). 